\author{
Original
}

\title{
Multi-resolution Laws' Masks based texture classification
}

\author{
Sonali Dash*, Uma Ranjan Jena \\ Department of Electronics and Telecommunication Engineering, Veer Surendra Sai University of Technology (VSSUT), Burla 768018, Odisha, India
}

Received 4 December 2015; accepted 16 July 2017

Available online 3 January 2018

\begin{abstract}
Wavelet transforms are widely used for texture feature extraction. For dyadic transform, frequency splitting is coarse and the orientation selection is even poorer. Laws' mask is a traditional technique for extraction of texture feature whose main approach is towards filtering of images with five types of masks, namely level, edge, spot, ripple, and wave. With each combination of these masks, it gives discriminative information. A new approach for texture classification based on the combination of dyadic wavelet transform with different wavelet basis functions and Laws' masks named as Multi-resolution Laws' Masks (MRLM) is proposed in this paper to further improve the performance of Laws' mask descriptor. A $k$-Nearest Neighbor $(k-\mathrm{NN})$ classifier is employed to classify each texture into appropriate class. Two challenging databases Brodatz and VisTex are used for the evaluation of the proposed method. Extensive experiments show that the Multi-resolution Laws' Masks can achieve better classification accuracy than existing dyadic wavelet transform and Laws' masks methods.

(C) 2017 Universidad Nacional Autónoma de México, Centro de Ciencias Aplicadas y Desarrollo Tecnológico. This is an open access article under the CC BY-NC-ND license (http://creativecommons.org/licenses/by-nc-nd/4.0/).
\end{abstract}

Keywords: Multi-resolution Laws’ Masks; Dyadic wavelet transform; Feature extraction; Texture classification

\section{Introduction}

Texture is a repetition of intensity patterns present in an image. It is one of the significant visual characteristic and a rich source of information for any image analysis. Hence, it is a vital element in many areas of applications, such as object recognition, content-based image retrieval, remote sensing, image segmentation, medical image analysis, automated inspection, document processing and so on. An important step in the classification of texture is the extraction of feature. The different types of feature extraction techniques for texture identification are structural (Haralick \& Shapiro, 1992), statistical (Haralick, 1979), geometrical (Pikaz \& Averbuch, 1997), model-based (Mao \& Jain, 1992) and signal processing (Laine \& Fan, 1993). Gaussian Markov random fields (GMRF) and Gibbs random fields are also proposed to characterize textures (Cross \& Jain, 1983; Kashyap \& Khotanzed, 1986). In addition, local linear

\footnotetext{
* Corresponding author.

E-mail address: sonali.isan@gmail.com (S. Dash).
}

Peer Review under the responsibility of Universidad Nacional Autónoma de México. transformations are utilized to compute texture (Laws, 1980a; Unser, 1986). The traditional statistical approaches for texture analysis have limitations to spatial interactions over small neighbourhoods on a single scale only.

In the field of multi-resolution analysis, wavelet analysis has become a powerful tool during the past decades. The wavelet transformation provides a precise and unifying framework for the analysis and characterization of signals at different scales. The discrete wavelet transform of an image simultaneously reserves the time and frequency domain information of the signal and efficiently extracts the key features of the image. Several multi-resolution and multi-channel transform algorithms have been used for texture classification such as dyadic wavelet transform (Arivazhagan \& Ganesan, 2003; Mallat, 1989a, 1989b), wavelet frame transform (Unser, 1995), Gabor filters (Jain \& Farrokhnia, 1991; Manjunath \& Ma, 1996), steerable pyramids (Simoncelli \& Freeman, 1995) and fusion of multi-resolution methods (Li \& Shawe-Taylor, 2005). The signal processing techniques rely mainly on texture filtering to analyze the frequency content either in spatial domain (Laws, 1980b; Unser, 1986) or in frequency domain (Bajcsy \& Lieberman, 1976). Among the multi-resolution techniques, discrete wavelet transform is not translation-invariant (Xiong, Zhang, \& Moon, 2000). 
Although the aforementioned methods proved to deliver good classification accuracy, recently some authors have introduced hybrid models jointly by combining the traditional techniques with multi-resolution techniques for an additional improvement of texture classification. Few examples of hybrid models proposed are Multi-scale grey level co-occurrence matrices for texture description, Multi-resolution LBP (Ojala, Pietikainen, \& Maenpaa, 2002), Gaussian image pyramid-based texture features and Multi-scale Co-occurrence Local Binary Pattern. (Siqueira, Schwartz, \& Pedrini, 2013; Yadav, Anand, Dewal, \& Gupta, 2015a, 2015b; Qi, Shen, Zhao, Li, \& Pietikainen, 2015).

The Laws' masks awareness also known as energy filters as conveyed by K.I. Laws, where he has suggested towards the filtering of images with specific masks created from the combination of one-dimensional kernel vector in order to assess the texture properties (Laws, 1979, 1980a). In the medical image analysis, Laws' masks have received wide acceptance (Elnemr, 2013; Rachidi et al., 2008) but for texture classification, Laws' masks have resulted very poor classification accuracy. Pietikainen has found that the performance of texture energy measures depends on the general forms of the masks rather than on their specific values (Pietikainen, Rosenfled, \& Davis, 1983). To find the local rank order correlations of images with appropriate image and mask sizes, Laws' masks could achieve better result than achieved by the basic convolutions as reported by Harwood (Harwood, Subbarao, \& Davis, 1985). Ade has considered different methodologies associated with Laws' measures while characterizing textures by using Eigenfilters (Ade, 1983). Comparative studies carried out by Buf and $\mathrm{Ng}$ have reported that Laws, Haralick and Unser methods provide better results for texture features (Buf, Kardan, \& Spann 1990; Ng, Tan, \& Kittler, 1992). In 1995, Harwood et al. have suggested a new method of texture analysis based on a local centre-symmetric covariance analysis, using Kullback (log-likelihood) discrimination of sample and prototype distribution (Harwood, Ojala, Pietikainen, Kelman, \& Davis, 1995). This method can be viewed as a generalization of Laws' approach and the results are compared with that of Laws' measures. The experiments are carried out with 24 different Laws' $(5 \times 5)$ masks, and the classification error rates of $25.9 \%$ (image size of $64 \times 64$ ) and $39.2 \%$ (image size of $32 \times 32$ ) are achieved on Brodatz database. Sharma and Singh have shown maximum classification accuracy of $83.30 \%$ by using Laws' mask method for MeasTex database with $k$-NN as classifier (Sharma \& Singh, 2001). The same authors Singh and Sharma have done another experiment with MeasTex and VisTex benchmarks with different texture descriptors. They have stated that by utilizing Laws' mask descriptor, the classification accuracies for MeasTex database are $82.80 \%$ with linear classifier, $75.10 \%$ with $k$-NN as classifier on original data and $69.30 \%$ with $k$-NN as classifier on PCA data. The classification accuracies for VisTex database are $68.80 \%$ with linear classifier, $56.10 \%$ with $k$-NN as classifier on original data and $53.20 \%$ with $k$-NN as classifier on PCA data (Singh \& Sharma, 2001). Ertugrul has described adaptive texture energy measure. By employing adaptive texture energy measure best classification accuracy of $68.43 \%$ is obtained on Brodatz database (Ertugrul, 2014).
As we have examined our proposed method with Brodatz and VisTex database, a brief review on recently developed methods that classify these two databases are discussed. Qiao et al. have proposed a new method for extraction of texture features with a combination of the phase difference and the magnitude variation information of the complex wavelet coefficients, and then modelled the measure with the real generalized Gaussian distribution (GGD). They have verified the proposed method with two different datasets created from Brodatz database with different sizes of images and the third dataset is created from VisTex database with $k$-NN as classifier. The best classification accuracies achieved are $92.1 \%$ on the first dataset of Brodatz and $83.1 \%$ on the second dataset of Brodatz. Classification accuracy of $84.6 \%$ is obtained on VisTex database (Qiao, Zhao, \& Song, 2009). Dong and Ma have suggested a new method for feature extraction through contourlet subband clustering for texture classification. They have evaluated the proposed method with two different datasets created from Brodatz database and two different datasets created from VisTex database, and obtained different classification results from each created databases using $k$-NN as classifier. They have achieved classification accuracy of $99.92 \%$ on the first dataset and $96.81 \pm 0.44 \%$ on the second dataset of Brodatz database. The best classification accuracy of $99.25 \pm 0.38 \%$ is obtained on the first dataset and $85.95 \pm 1.50 \%$ on the second dataset of VisTex database (Dong \& Ma, 2013). Susan and Hanmandlu have proposed a new method of probabilistic non-extensive entropy feature for texture characterization and achieved highest classification accuracy of $97.78 \%$ on Brodatz database using SVM as classifier (Susan \& Hanmandlu, 2013). Yuan has recommended a rotation and scale invariant local binary pattern by collectively considering high order directional derivatives, circular shift sub-uniform, and scale space. The proposed method delivered highest classification accuracy of $75.2 \%$ on Brodatz database (Yuan, 2014). Murala and Wu have proposed a combination of Gabor transform with robust local binary (RLBP) operator and the maximum classification accuracy is found to be $84.92 \%$ on Brodatz database and $93.25 \%$ on VisTex database (Murala \& Wu, 2014). Zhang et al. have recommended a technique by combining spiking neural network and Fast wavelet transform (SNN-FWT), and obtained best classification rate of $98.93 \%$ on Brodatz database with $k$-NN as classifier (Zhang, Wu, Zhuo, Wang, \& Huang, 2015). Florindo et al. have recommended combinations of traditional texture descriptors with non-additive entropy. They have achieved best classification accuracy of $92.51 \%$ on Brodatz database and $93.52 \%$ on VisTex database with a combination of grey level co-occurrence matrix (GLCM) with non-additive entropy using Linear Discriminant Analysis (LDA) as classifier (Florindo, Assirati, \& Bruno 2015). Tang et al. have suggested a new texture extraction method combining non-overlap window local binary pattern and grey level co-occurrence for green tea leaves classification, where they have also verified the proposed method on Brodatz database. They have achieved maximum classification accuracy of $94.8 \%$ on Brodatz database with back propagation neural network as classifier (Tang et al., 2015).

A thorough literature review in texture classification concludes that Law's mask descriptor provides poor classifi- 
cation accuracy for texture classification (Ojala, Pietikainen, \& Harwood, 1996; Ojala, Pietikainen, \& Nisula, 1996; Vidya, Ramón, \& Praveen, 2000). In addition, the study reveals that there has not been any further research for the improvement of texture classification by using Laws' mask method.

This paper presents a new approach for extending Laws' mask to multi-resolution representation. Two existing texture descriptors, dyadic wavelet transform (DWT) and Laws' mask are integrated for the extension and that is named as Multi-resolution Laws' Masks (MRLM) method. The proposal is assessed by comparing the performance of the classical approaches with MRLM approach. Two benchmark texture datasets: Brodatz and VisTex are considered to verify the effectiveness of the proposed method. Simple $k$-nearest neighbor $(k-\mathrm{NN})$ classifier is employed for texture classification. The experiments carried out in this paper are as follows:

a. The first experiment deals with the performance evaluation of the traditional DWT method. DWT with different wavelet basis functions (db1, db4, db8, coif1, coif4, sym2) are applied on two sets of texture databases. Then, statistical features such as mean and standard deviation are extracted from the decomposed images for different levels.

b. The second experiment deals with the performance evaluation of the traditional Laws' mask method. The Laws' mask descriptors having 15 numbers of masks and 25 numbers of masks are directly applied to the texture images of two datasets. The output results are then passed through three different types of energy measurement filters. Then after normalization, statistical features like absolute mean, mean square and entropy are extracted from different energy measurement filters.

c. The third experiment evaluates the effectiveness of the proposed MRLM method. DWT with different wavelet basis functions (db1, db4, db8, coif1, coif4, sym2) are applied on two sets of texture databases. Then, Laws' masks descriptors are applied on the decomposed images obtained at different levels with different wavelet functions. The output results are then passed through three different types of energy measurement filters. After normalization, statistical features like absolute mean, mean square and entropy are extracted from different energy measurement filters.

The results show that the MRLM approach is capable of providing higher classification accuracy than the traditional DWT and Laws' masks methods for both the databases. The major steps involved to accomplish the process are texture feature extraction, feature normalization and classification.

The organization of this paper is as follows: Section 2 takes a brief review of 2D-DWT and Laws' masks. Section 3 describes the proposed method. Section 4 presents details of experimental results. Conclusions are drawn in section 5 .

\section{Review of dyadic wavelet transform and Laws' Masks method}

\subsection{Dyadic wavelet transform}

The 2D-DWT is a multi-resolution analysis and decomposes images into wavelet coefficients and scaling functions. Dyadic wavelet decomposition is achieved using 2 channel filter banks composed of a low-pass $(\mathrm{G})$ and high-pass (H) filter and each filter bank is then sampled at a half rate of the previous frequency. DWT transforms the original image into four subimages namely low-low (LL), low-high (LH), high-low (HL) and highhigh $(\mathrm{HH})$ through low pass filters and high pass filters. An example of DWT with two levels of decomposition is shown in Figure 1. Every subimage contains information of a specific scale and orientation. The original image is thus represented by a set of subimages at several scales for which they are called the multi-scale representation. Because the images are dilated by a factor of two, this is also known as dyadic wavelet transform. The features obtained from these DWT transformed images are useful for texture analysis. A number of basis functions are used as the mother wavelet for wavelet transformation. The mother wavelet determines the characteristics of the resulting wavelet transformation. Some of the wavelet functions that are used for wavelet transformation are Haar, Daubechies, Symlet, Coiflets, Bior, Meyer, Morlet, etc. The oldest and the simplest wavelet is the Haar wavelet. The Daubechies wavelets are the most popular wavelets. The Haar, Daubechies, Symlet and Coiflets are compactly supported orthogonal wavelets. The wavelets are selected on the basis of their shape and ability to analyze the signal in particular application.

\subsection{Laws' masks}

Laws observed that certain gradient operator such as Laplacian and Sobel operators accentuate the underlying microstructure of texture within an image. This is the foundation for a feature extraction scheme where a series of pixel impulse response arrays are obtained from combinations of 1-D vectors. Laws' properties, which he called "texture energy measures", are extracted from three simple vectors of length $3, \mathrm{~L} 3 \equiv(1,2$, $1), \mathrm{E} 3 \equiv(-1,0,1)$, and $\mathrm{S} 3 \equiv(-1,2,-1)$, which represent the one-dimensional operations of centre-weighted local averaging, symmetric first differencing (edge detection), and second differencing (spot detection) respectively. If we convolve these vectors with themselves or each other, we obtain five vectors of length 5:

$\mathrm{L} 5 \equiv(1,4,6,4,1)=\mathrm{L} 3 * \mathrm{~L} 3, \mathrm{~S} 5 \equiv(-1,0,2,0,-1)=-\mathrm{E} 3 * \mathrm{E} 3=$ $\mathrm{L} 3 * \mathrm{~S} 3, \quad \mathrm{R} 5 \equiv(1,-4,6,-4,1)=\mathrm{S} 3 * \mathrm{~S} 3, \quad \mathrm{E} 5 \equiv(-1,-2,0,2,1)=$ $\mathrm{L} 3 * \mathrm{E} 3$ and $\mathrm{W} 5 \equiv(-1,2,0,-2,1)=-\mathrm{E} 3 * \mathrm{~S} 3$. Sets of more number of vectors can be defined by repeating this convolution process. Therefore, there are five types of masks namely Edge (E), Level (L), Spot (S), Ripple (R) and Wave (W). From these set of masks 25 different masks are produced. These masks are subsequently convolved with a texture image to accentuate their microstructure giving an image from which the energy of the microstructure arrays is measured together with other statistics. 


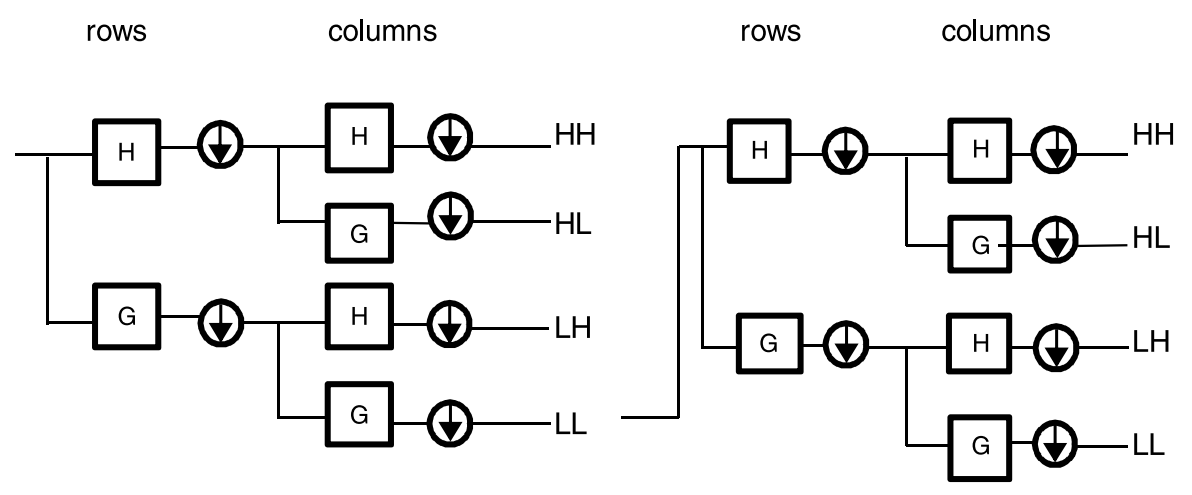

Fig. 1. Two-level DWT.

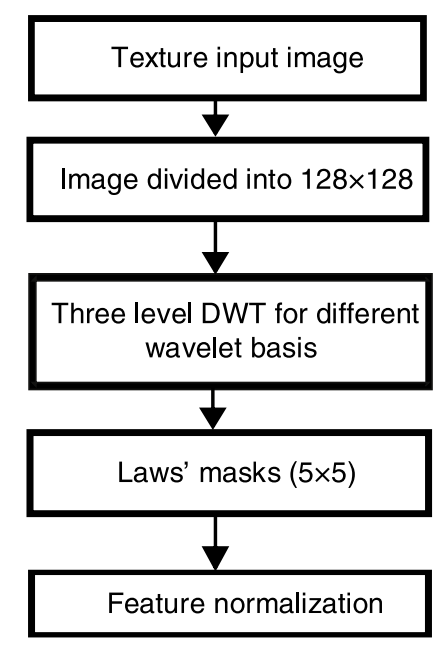

Fig. 2. Block diagram of proposed method.

\section{Proposed Multi-resolution Laws' Masks (MRLM) method}

The prime objective of this investigation is to assess the performance of the proposed MRLM technique for extraction of texture features by integrating DWT and Laws' mask, the two existing texture descriptors. The texture features are classified by $k$-NN classifier. MRLM method is introduced for further enhancement of classification accuracy than the traditional dyadic wavelet transform and Laws' masks methods. The procedural steps of the proposed method are given in Figure 2.

In the first step called the pre-processing step, database is generated by splitting of the original image into subimages. In the second step, texture images are decomposed through DWT up to three levels by incorporating different wavelets like Daubechies (db1, db4, db8), Coif (coif1, coif4) and Symlet (sym2). The decomposition process divides the grey scale image into four identical subbands, viz., approximation (LL), horizontal (LH), vertical (HL) and diagonal (HH) components of equal size at the first level of decomposition. Most of the image energy is concentrated in the low frequency subband LL. On the other hand, the high frequency subbands $\mathrm{LH}, \mathrm{HL}$ and $\mathrm{HH}$ contain edges and texture of the images. Then, LL component is further decomposed into four equal sizes of subimages (LL1, LH1, HL1, HH1) at the second level of decomposition. This procedure of the subdividing is repeated until the third level of decomposition is reached. We select all the subbands from each decomposition level and the resulted transformed images of all the subbands are used for further analysis by employing Laws' masks method. In the third step, the transformed images obtained from the four subbands are convolved with Laws' masks. There are five types of masks, as mentioned earlier: Level (L), Edge (E), Spot (S), Ripple (R) and Wave (W). Thereafter the convolved outputs are passed through texture energy measurement filters. The convolved outputs are passed through three types of energy measurement filters (Elnemr, 2013), which are described as follows:

$\mu=\frac{\sum_{N} N_{p}}{N}$

$\mu_{a b s}=\frac{a b s\left(\sum_{N} N_{p}\right)}{N}$

$\sigma=\sqrt{\frac{\sum_{N}\left(N_{p}-\mu\right)^{2}}{N}}$

where $\mu$ presents the mean filter, $\mu_{a b s}$ presents the absolute mean filter, $\sigma$ presents the standard deviation filter, $N_{p}$ presents the neighbouring pixels and $N$ presents the window size. The texture feature vectors acquired from each energy measurement filters resulting different ranges of values, which are normalized by min-max normalization method, thus contributing it in the form that is applicable as an input to the classifier. For each normalized feature vector, we evaluated three most important statistical features that are represented as textural parameters: absolute mean (ABSM), mean square or energy (MS) and entropy. Three statistical features are calculated for individual energy measurement filter.

\section{Experimental results, comparison and discussion}

The prime motivation of this section is to investigate the proposed MRLM method along with the conventional DWT and Laws' masks methods to extract texture features for texture classification. To illustrate the efficiency of the proposed method the results are compared with the existing Laws' masks method. The performance of conventional dyadic wavelet transform, Laws' masks and proposed MRLM methods are examined by using Brodatz and VisTex databases (Brodatz, 1966; VisTex, 1995). 


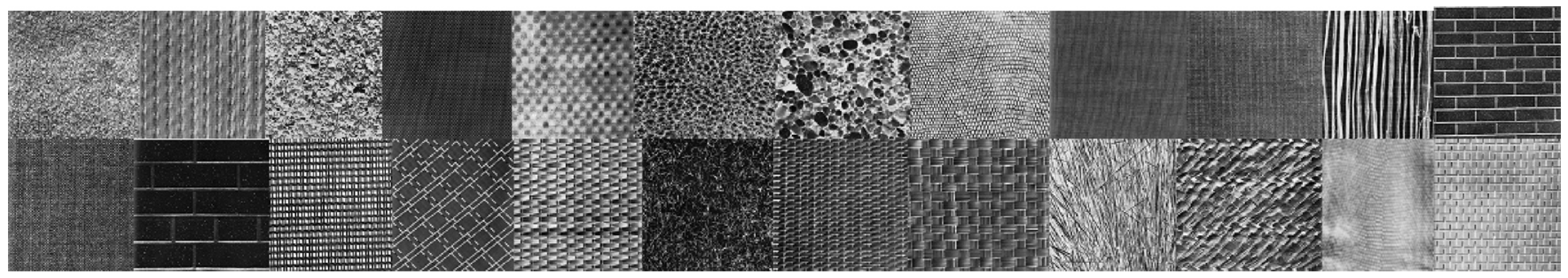

Fig. 3. 24 classes of Brodatz texture.
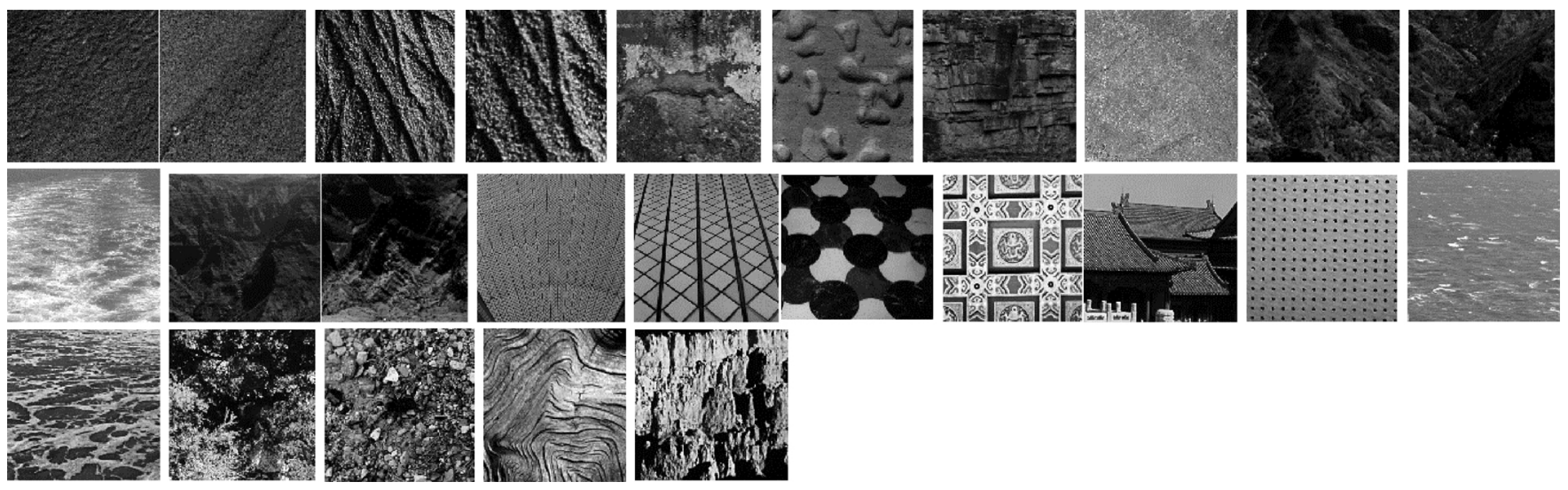

Fig. 4. 25 classes of VisTex texture.

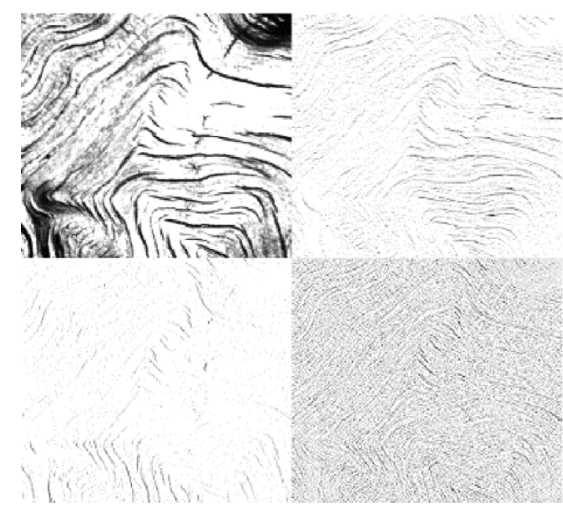

First level decomposition,

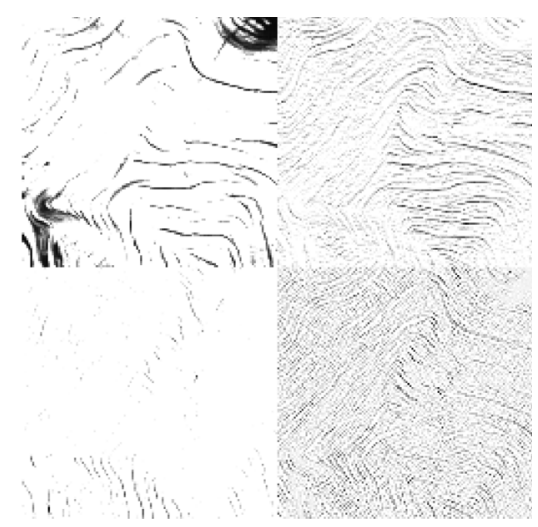

Second level decomposition.

Fig. 5. First level decomposition, second level decomposition.

The Brodatz album is a widely used database and often treated as a benchmark for texture classification. There are substantial variations within some textures and some of them are so alike to each other that is why this Brodatz dataset is very challenging. The first database consists of 24 texture images each of size $640 \times 640$ pixels from the Brodatz database. Figure 3 represents the sample images of Brodatz database. In the experiment, each image is divided into 25 nonoverlapping samples of size $128 \times 128$ pixels. Therefore, there are 600 subimages derived from selected 24 images. Out of 25 subimages of each texture image, we have taken 12 subimages for training and the remaining 13 subimages for testing. The Vision Texture (VisTex) dataset is prepared by the Massachusetts Institute of Technology (MIT). VisTex database contains colour texture images that represent the real world conditions. Our second database includes
25 texture images of size $512 \times 512$ pixels from VisTex database and split into 16 nonoverlapping samples of size $128 \times 128$ pixels. Thus there are total 400 samples available out of which 200 samples are used for training and 200 samples for testing. The images are converted to grayscale before feature extraction. Figure 4 depicts the sample images of VisTex database. The achievement of classification success rate is verified using $k$-NN classifier.

In our first experiment, we use the traditional DWT where the training and testing images are decomposed to three levels by using $\mathrm{db} 1, \mathrm{db} 4, \mathrm{db} 8$, coif 1 , coif 4 and sym 2 as basis functions. The transformed images obtained from first and second level of decompositions are shown in Figure 5. When the original image is decomposed to first level of decomposition by applying any one of the wavelets, four subbands LL, LH, HL 
Table 1

Classification results for traditional dyadic wavelet transform.

\begin{tabular}{|c|c|c|c|}
\hline Different wavelets & $\begin{array}{l}\text { Number of } \\
\text { features }\end{array}$ & $\begin{array}{l}\text { Classification } \\
\text { accuracy }(\%) \text { for } \\
\text { Brodatz dataset }\end{array}$ & $\begin{array}{l}\text { Classification } \\
\text { accuracy }(\%) \text { for } \\
\text { VisTex dataset }\end{array}$ \\
\hline DWT (db1, $L=3)$ & 24 & 90.71 & 48.50 \\
\hline DWT (db4, $L=3$ ) & 24 & 90.38 & 49.50 \\
\hline DWT (db8, $L=3)$ & 24 & 90.38 & 44.50 \\
\hline $\begin{array}{l}\text { DWT (coif1, } \\
\qquad L=3 \text { ) }\end{array}$ & 24 & 90.38 & 49.50 \\
\hline $\begin{array}{l}\text { DWT (coif4, } \\
\quad L=3 \text { ) }\end{array}$ & 24 & 88.14 & 44.00 \\
\hline $\begin{array}{c}\text { DWT (sym2, } \\
\quad L=3)\end{array}$ & 24 & 89.10 & 50.50 \\
\hline
\end{tabular}

and $\mathrm{HH}$ are obtained. We select all the subbands from each decomposition level. Then statistical texture features like mean and standard deviation are extracted from the detail regions of DWT decomposed images, at different scale. Hence, in total eight numbers of statistical features are derived from the first level decomposition of one image. Accordingly, for three levels of decomposition, total 24 features are extracted from each image. In the similar way, all the features are derived by applying different wavelets individually to both the databases. A $k$-NN classifier is used to classify each texture feature to an appropriate class. The classification results of the conventional dyadic wavelet transform for all the basis functions are tabulated in Table 1 and shown in Figure 6. From Table 1, it is observed that DWT with db1 as basis achieves the highest accuracy of $90.71 \%$ on Brodatz database. DWT with sym 2 as basis achieves the best classification accuracy of $50.50 \%$ on VisTex database.

In the second experiment, we use the conventional Laws' masks method in two ways. Initially only four masks Level (L), Edge (E), Spot (S) and Ripple (R) are used. Fifteen different masks are produced by using these four masks. Secondly, we use all the five masks Level (L), Edge (E), Spot (S), Ripple $(\mathrm{R})$ and Wave $(\mathrm{W})$, and twenty five different masks are produced by utilizing these five masks. Both training and testing images are convolved with these fifteen and twenty five numbers of masks corresponding to our two methods. The convolved outputs are passed through three energy measurement filters of size $15 \times 15$, which are normalized by min-max normalization method and then the statistical features Absolute mean, Mean square or energy and entropy are calculated, and these are used as texture features. For fifteen numbers of masks 45 numbers

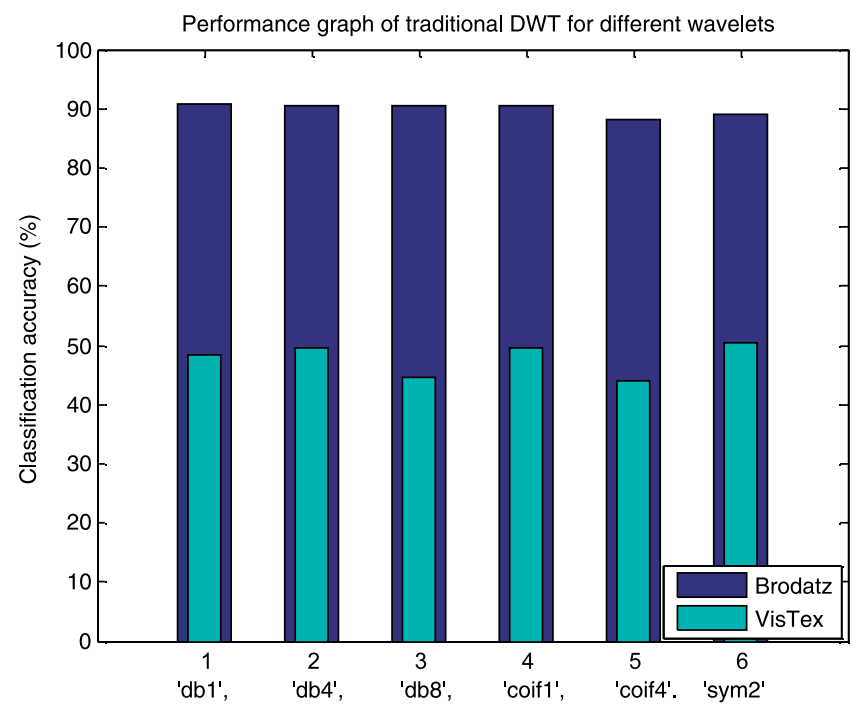

Fig. 6. Performance results of only DWT with different wavelet functions.

Performance of laws' masks for brodatz dataset

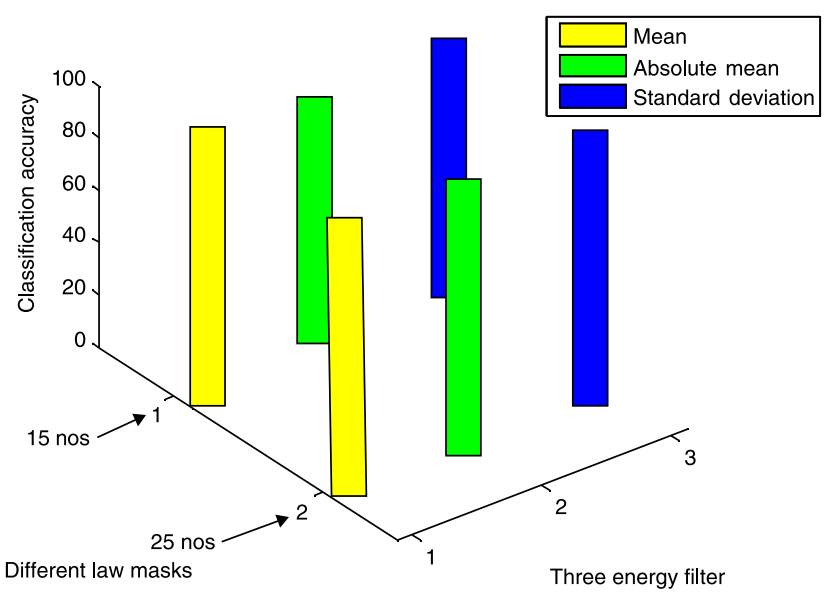

Fig. 7. Laws' mask for Brodatz dataset.

and for twenty five numbers of masks 75 numbers of features are extracted for each image. The classification results for both the databases are presented in Table 2. The performance results for both the datasets are shown in Figures 7 and 8. From Table 2, it is observed that Laws' masks of 25 numbers for the mean filter have produced the highest classification accuracy of $95.83 \%$ on Brodatz dataset and accuracy of $59.00 \%$ on VisTex dataset.

Table 2

Classification results for conventional Laws' masks $(5 \times 5)$.

\begin{tabular}{|c|c|c|c|c|}
\hline \multirow[t]{2}{*}{ Different size of masks } & \multirow[t]{2}{*}{ No. of features } & \multicolumn{3}{|c|}{ Classification accuracy } \\
\hline & & Mean & Absolute mean & Standard deviation \\
\hline \multicolumn{5}{|l|}{ Brodatz dataset } \\
\hline 15 number of masks (without wave) & 45 & 93.27 & 86.22 & 89.42 \\
\hline 25 number of masks & 75 & 95.83 & 91.35 & 91.67 \\
\hline \multicolumn{5}{|l|}{ VisTex dataset } \\
\hline 15 number of masks (without wave) & 45 & 57.00 & 48.00 & 53.00 \\
\hline 25 number of masks & 75 & 59.00 & 52.50 & 55.00 \\
\hline
\end{tabular}


Performance of laws' masks for vist ex dataset

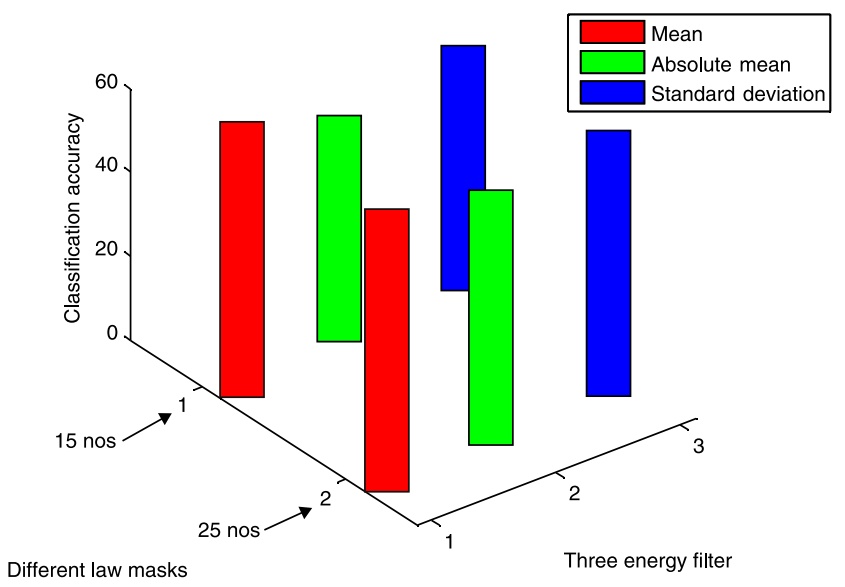

Fig. 8. Laws' mask for VisTex dataset.

Laws' masks with 15 numbers for the mean filter have produced highest classification accuracy of $93.27 \%$ on Brodatz dataset and accuracy of $57.00 \%$ on VisTex dataset.

The third experiment is our proposed MRLM method. By applying DWT on texture images for three levels of decomposition with different wavelets the transformed images are obtained for all the four sub bands LL, LH, HL and HH. These transformed images are further applied to Laws' masks descriptor for feature extraction and classification. As described in the second experiment that 15 numbers of Laws' masks will produce 45 features for each image. Hence, after convolving with the first level of decomposed images it will produce 180 features for each image. Similarly, 360 features for the second level decomposition and 540 features for the third level decomposition are obtained for 15 numbers of Laws' masks. In the same way 300, 600 and 900 features are derived from each image for first, second and third level of decompositions respectively for 25 numbers of Laws' masks. Classification accuracies are verified for both 15 and 25 numbers of different masks.
From Table 3, we can observe that for the Brodatz database, MRLM method for mean filter with $\mathrm{db} 1$ and sym 2 as basis have been able to achieve highest classification accuracy of $95.83 \%$ at the first level of decomposition. The second best classification accuracy of $95.51 \%$ is obtained from the same mean filter having coif1 as wavelet basis. Also for db8 classification accuracy of $93.91 \%$ is obtained which is higher than the traditional Laws' mask descriptor. For coif4 accuracy of $93.27 \%$ is achieved which is same as traditional Laws' mask method. Furthermore, for absolute mean and standard deviation filter it has detected that classification accuracies of $86.54 \%$ and $90.06 \%$ for $\mathrm{db} 1$ wavelet, which shows better accuracy than traditional Laws' masks method. The lowest classification accuracy of $92.31 \%$ is obtained for the mean filter with $\mathrm{db} 4$ as basis. The classification results for the higher levels of decomposition are listed in Tables 4 and 5 respectively, which show that the classification accuracies are reduced for the mean and absolute mean filters. However, it is noticed that the standard deviation filter has yielded an accuracy of $90.71 \%$ at second and third level of decomposition with coiflas basis. Figure 9 shows the performance results of Brodatz database for the proposed MRLM method with 15 numbers of masks.

The results obtained for VisTex database for the first level of decomposition are also given in the same Table 3. It can be seen that the proposed method gives the best accuracy of $63 \%$ with coif 4 as basis. The second and third best accuracy of $62 \%$ and $61 \%$ are achieved from sym 2 and coif 1 as wavelets basis respectively. The wavelets $\mathrm{db} 1$ and $\mathrm{db} 4$ provide an accuracy of $60 \%$. The lowest accuracy of $58.50 \%$ is obtained with $\mathrm{db} 8$ as basis, but still it is higher than the existing descriptors. From Table 4 it is observed that the highest classification accuracy of $66 \%$ is obtained at the second level of decomposition with db8 as basis, which is $9 \%$ more than the existing Laws' masks descriptor. In addition, we have achieved better accuracy for other wavelet basis functions such as, $64.50 \%$ for $\mathrm{db} 4$ and coif $4,61.50 \%$ for sym2, $61 \%$ for $\mathrm{db} 1$ and $60.50 \%$ for coif 1 as listed in Table 4. At the third level of decomposition, the

Table 3

Classification results for one level Multi-resolution Laws' Masks (MRLM) for 15 number of different masks.

\begin{tabular}{|c|c|c|c|c|}
\hline \multirow[t]{2}{*}{ MRLM with different wavelet basis } & \multirow[t]{2}{*}{ Number of features } & \multicolumn{3}{|c|}{ Classification accuracy } \\
\hline & & Mean & Absolute mean & Standard deviation \\
\hline \multicolumn{5}{|l|}{ Brodatz dataset } \\
\hline $\operatorname{MRLM}(\mathrm{db} 1, L=1)$ & 180 & 95.83 & 86.54 & 90.06 \\
\hline $\operatorname{MRLM}(\mathrm{db} 4, L=1)$ & 180 & 92.31 & 81.41 & 86.41 \\
\hline $\operatorname{MRLM}(\mathrm{db} 8, L=1)$ & 180 & 93.91 & 83.01 & 85.58 \\
\hline MRLM (coif $1, L=1)$ & 180 & 95.51 & 86.57 & 88.78 \\
\hline MRLM (coif $4, L=1)$ & 180 & 93.27 & 78.85 & 85.58 \\
\hline $\operatorname{MRLM}(\operatorname{sym} 2, L=1)$ & 180 & 95.83 & 85.90 & 88.78 \\
\hline \multicolumn{5}{|l|}{ VisTex dataset } \\
\hline $\operatorname{MRLM}(\mathrm{db} 1, L=1)$ & 180 & 60.00 & 42.50 & 42.50 \\
\hline $\operatorname{MRLM}(\mathrm{db} 4, L=1)$ & 180 & 60.00 & 40.00 & 42.00 \\
\hline $\operatorname{MRLM}(\mathrm{db} 8, L=1)$ & 180 & 58.50 & 40.50 & 41.00 \\
\hline MRLM (coif $1, L=1)$ & 180 & 61.00 & 42.50 & 43.00 \\
\hline MRLM (coif4, $L=1)$ & 180 & 63.00 & 42.00 & 45.00 \\
\hline $\operatorname{MRLM}(\operatorname{sym} 2, L=1)$ & 180 & 62.00 & 40.00 & 40.00 \\
\hline
\end{tabular}

Better results are made bold. 
Table 4

Classification results for two level Multi-resolution Laws' Masks (MRLM) for 15 number of different masks.

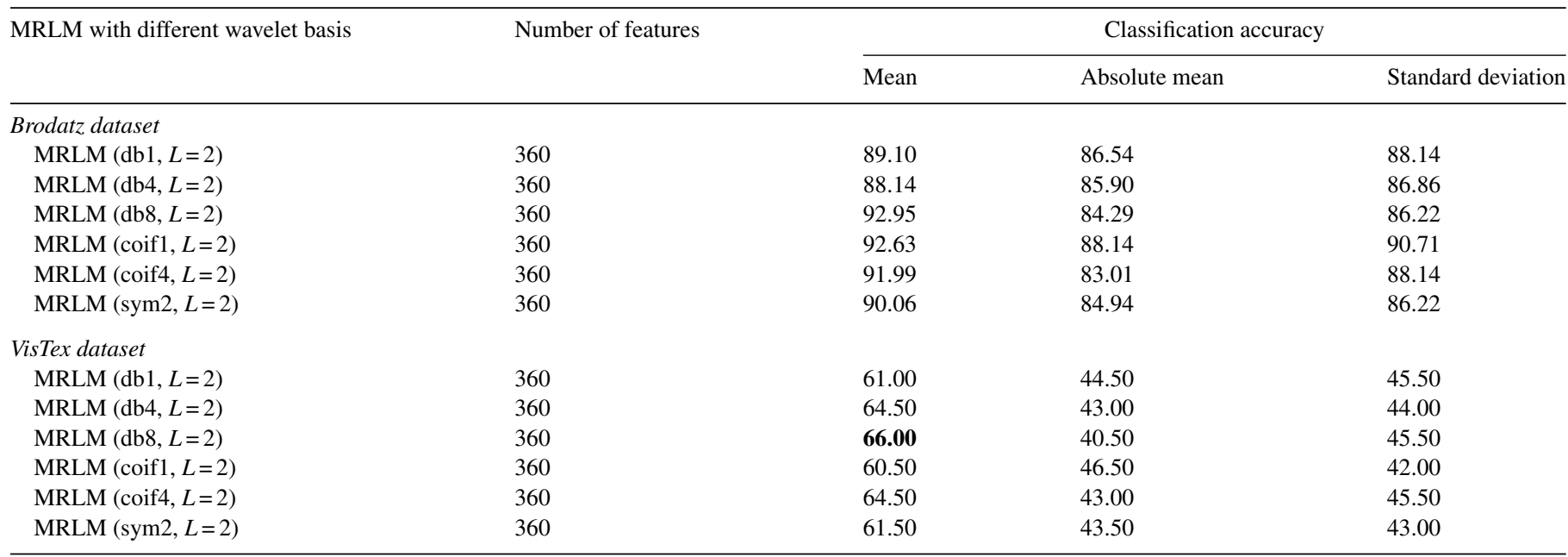

Better results are made bold.

Table 5

Classification results for three level Multi-resolution Laws' Masks (MRLM) for 15 number of different masks.

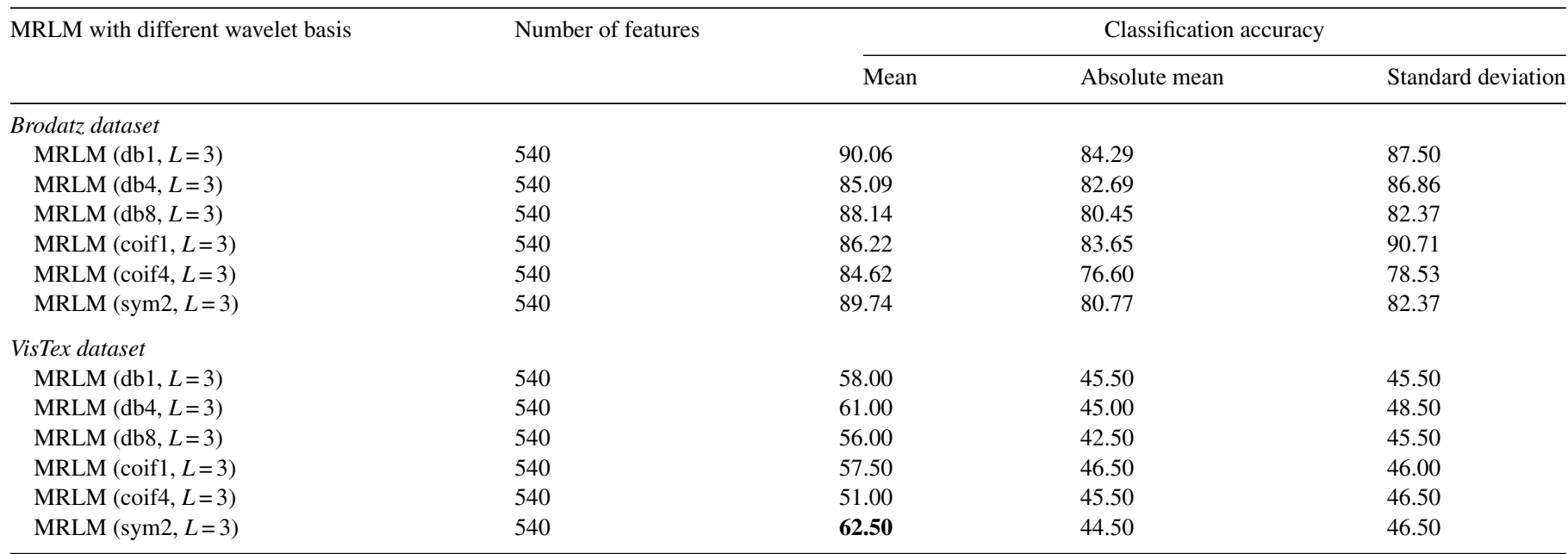

Better results are made bold.

Performance of first level MRLM method with 15 nos of mask for brodatz dataset

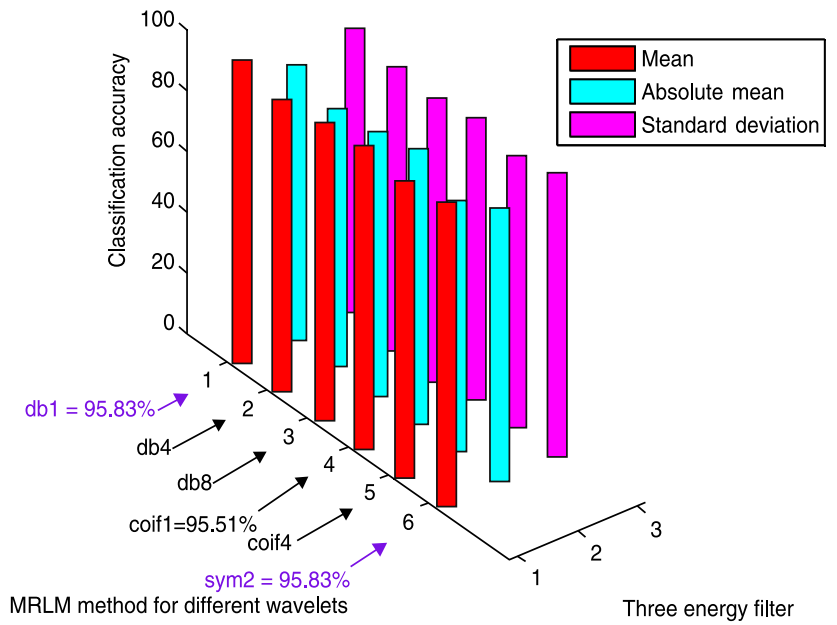

classification accuracy starts decreasing for some wavelet basis functions as reported in Table 5. The best classification accuracy of $62.50 \%$ is obtained with sym 2 as basis at the third level of decomposition. Accuracy of $61 \%$ for db4, $58 \%$ for $\mathrm{db} 1$ and $57.50 \%$ for coif 1 and the lowest accuracy of $56 \%$ for db8 and $51 \%$ for coif 4 are obtained at the third level of decomposition. The above mentioned results are achieved only for the mean energy filter. There is no improvement in classification accuracy for the absolute mean and standard deviation energy filters. Figures 11-13 show the performance results of VisTex database for the proposed MRLM method with 15 numbers of masks.

Table 6 exhibits the classification success rates of Brodatz database for the proposed method MRLM with 25 numbers of masks at the first level of decomposition. The results show that the highest classification accuracy $96.79 \%$ is achieved with $\mathrm{db} 1$ as basis for the mean filter. For absolute mean and standard deviation filters, classification accuracies of $89.74 \%$ and $92.95 \%$ are obtained respectively for the same wavelet basis. The

Fig. 9. MRLM (15 masks, $L=1$ ) for Brodatz dataset. 
Performance of first level MRLM method with 25 nos of mask for Brodatz dataset

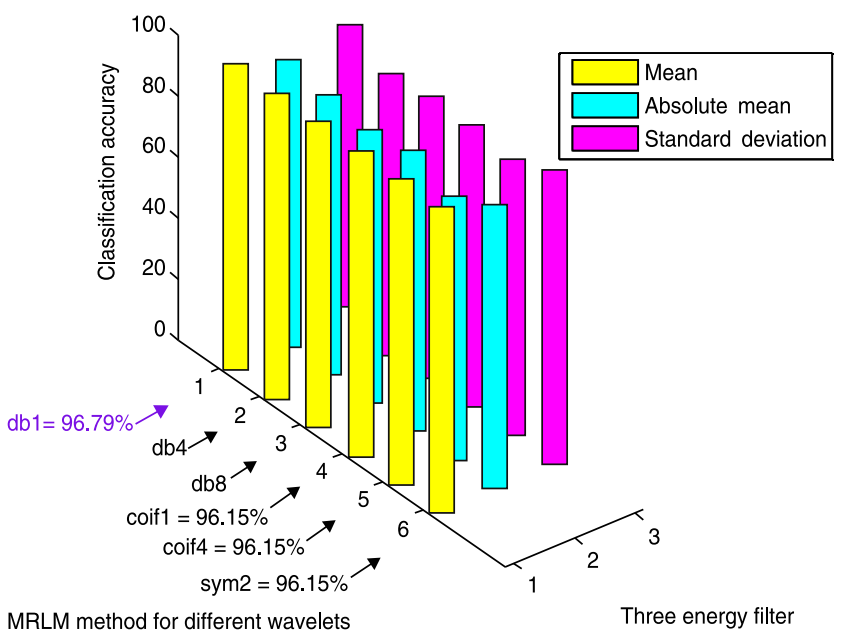

Fig. 10. MRLM (25 masks, $L=1)$ for Brodatz dataset.

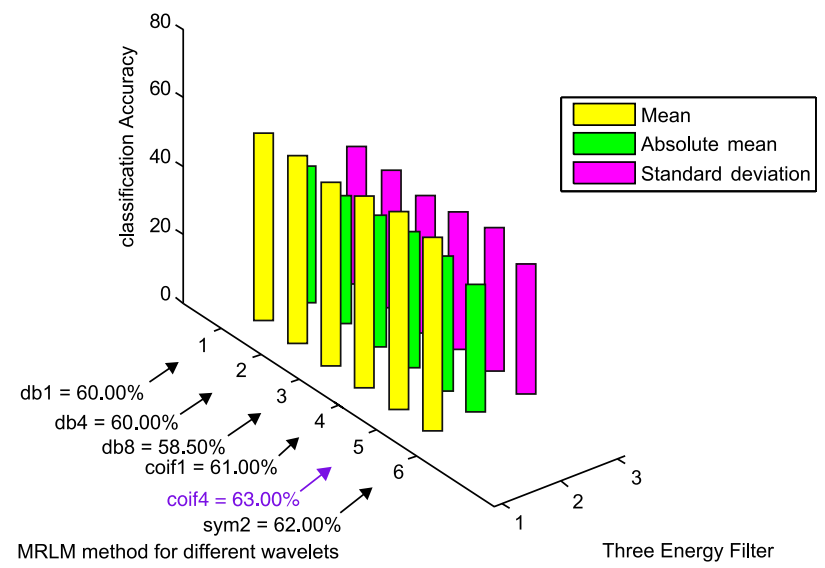

Fig. 11. MRLM (15 masks, $L=1$ ) for VisTex dataset.

Performance of second level MRLM method with 15 nos of mask for VisTex dataset

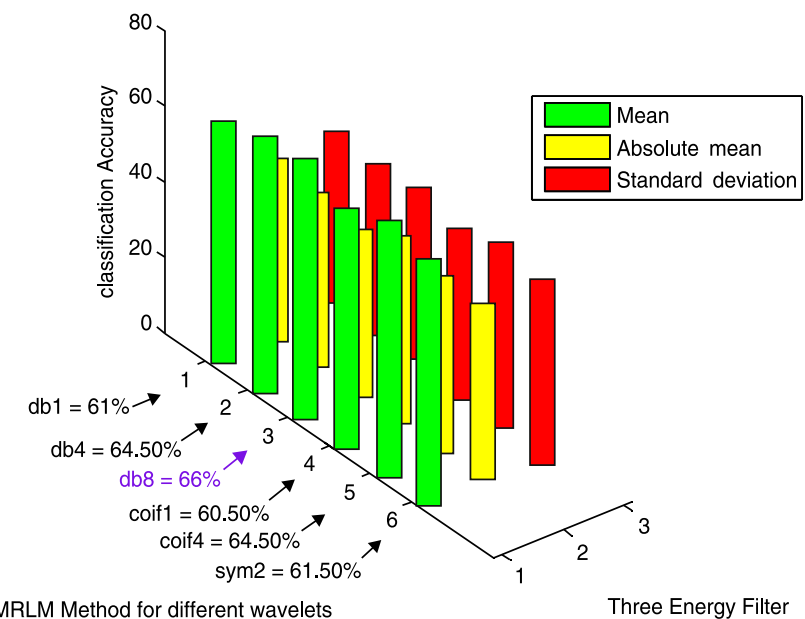

Fig. 12. MRLM (15 masks, $L=2$ ) for VisTex dataset.
Performance of third level MRLM method with 15 nos of mask for vistex dataset

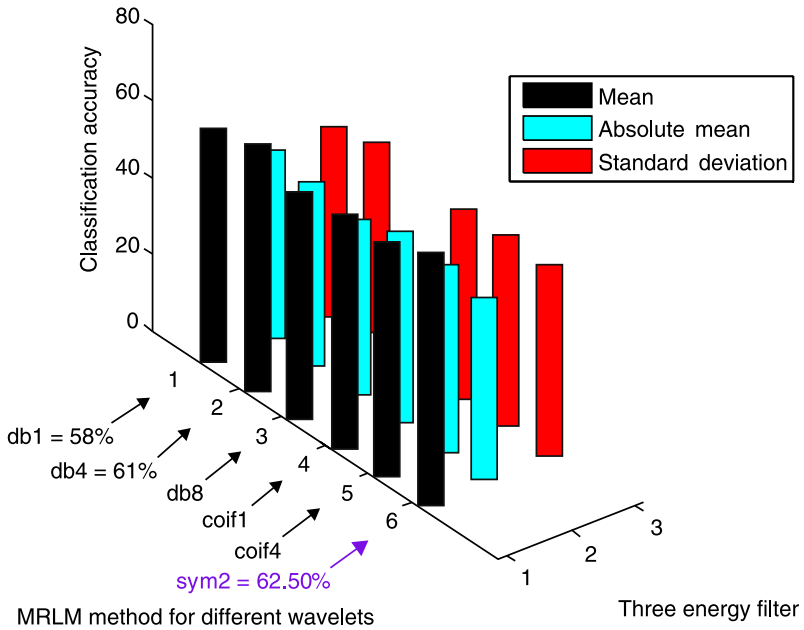

Fig. 13. MRLM (15 masks, $L=3$ ) for VisTex dataset.

second highest accuracy $96.15 \%$ is obtained from coif 1 , coif 4 and sym 2 as basis for the mean filter. The classification accuracy is reduced to $94.23 \%$ for $\mathrm{db} 4$ and $90.38 \%$ for db8 respectively for the mean filter. There is no further improvement of classification accuracy for the absolute mean and standard deviation filters, by using rest of all the wavelets for the first level of decomposition. Tables 7 and 8 display that at the higher levels of decomposition classification accuracies are reduced for the all the three energy measurement filters on Brodatz database. Figure 10 shows the performance results of Brodatz database for the proposed MRLM method with 25 numbers of masks.

The same Table 6 also shows the classification success rates for the proposed method MRLM with 25 numbers of masks at the first level of decomposition on VisTex database. At the first level of decomposition, the first best accuracy of $63.50 \%$ is obtained from db4 and coif 4 wavelets. The second best accuracy of $62.50 \%$ is achieved from sym 2 wavelet. Other wavelets also provide better accuracy results like, $62 \%$ with $\mathrm{db} 1$ and $\mathrm{db} 8$ as basis, $60 \%$ with coif 4 as basis. From Table 7 it can be seen that the highest classification accuracy of $67.50 \%$ is attained with $\mathrm{db} 8$ as basis at the second level of decomposition. Improvement in results are also noticed with other wavelets at the second level of decomposition such as, $65 \%$ for coif $4,64.50 \%$ for sym $2,63 \%$ for $\mathrm{db} 4,62.50 \%$ for $\mathrm{db} 1$ and $61 \%$ for coif 1 . The classification accuracies start decreasing at the third level of decomposition for some wavelets as reported in Table 8. The first best accuracy of $65 \%$ with sym 2 as basis is obtained at the third level of decomposition. The second best accuracy of $61 \%$ is achieved from $\mathrm{db} 4$ and $\mathrm{db} 8$ wavelets. The third highest accuracy of $60 \%$ is achieved with coif4 as basis. The lowest classification accuracies of 58\% from $\mathrm{db} 1$ and $57 \%$ from coif 4 are obtained at the third level of decomposition. Figures 14-16 show the performance results of VisTex database for the proposed MRLM method with 25 numbers of masks.

The experimental results show that the proposed MRLM method (for both 15 numbers and 25 numbers of masks) provides highest classification accuracy at the first level of decomposition 
Table 6

Classification results for one level Multi-resolution Laws' Masks (MRLM) for 25 number of different masks.

\begin{tabular}{|c|c|c|c|c|}
\hline \multirow[t]{2}{*}{ MRLM with different wavelet basis } & \multirow[t]{2}{*}{ Number of features } & \multicolumn{3}{|c|}{ Classification accuracy } \\
\hline & & Mean & Absolute mean & Standard deviation \\
\hline \multicolumn{5}{|l|}{ Brodatz dataset } \\
\hline $\operatorname{MRLM}(\mathrm{db} 1, L=1)$ & 300 & 96.79 & 89.74 & 92.95 \\
\hline $\operatorname{MRLM}(\mathrm{db} 4, L=1)$ & 300 & 94.23 & 87.50 & 86.54 \\
\hline $\operatorname{MRLM}(\mathrm{db} 8, L=1)$ & 300 & 90.38 & 85.58 & 87.82 \\
\hline MRLM (coif1, $L=1)$ & 300 & 96.15 & 87.82 & 88.14 \\
\hline MRLM (coif4, $L=1)$ & 300 & 96.15 & 82.37 & 85.98 \\
\hline MRLM (sym2, $L=1)$ & 300 & 96.15 & 88.46 & 91.90 \\
\hline \multicolumn{5}{|l|}{ VisTex dataset } \\
\hline $\operatorname{MRLM}(\mathrm{db} 1, L=1)$ & 300 & 62.00 & 43.00 & 41.00 \\
\hline $\operatorname{MRLM}(\mathrm{db} 4, L=1)$ & 300 & 63.50 & 44.00 & 46.00 \\
\hline $\operatorname{MRLM}(\mathrm{db} 8, L=1)$ & 300 & 62.00 & 43.00 & 41.50 \\
\hline MRLM (coif1, $L=1)$ & 300 & 60.00 & 41.00 & 43.50 \\
\hline MRLM (coif4, $L=1)$ & 300 & 63.50 & 46.00 & 46.50 \\
\hline $\operatorname{MRLM}(\operatorname{sym} 2, L=1)$ & 300 & 62.50 & 42.00 & 42.00 \\
\hline
\end{tabular}

Better results are made bold.

Table 7

Classification results for two level Multi-resolution Laws' Masks (MRLM) for 25 number of different masks.

\begin{tabular}{|c|c|c|c|c|}
\hline \multirow[t]{2}{*}{ MRLM with different wavelet basis } & \multirow[t]{2}{*}{ Number of features } & \multicolumn{3}{|c|}{ Classification accuracy } \\
\hline & & Mean & Absolute mean & Standard deviation \\
\hline \multicolumn{5}{|l|}{ Brodatz dataset } \\
\hline $\operatorname{MRLM}(\mathrm{db} 4, L=2)$ & 600 & 89.74 & 85.90 & 89.74 \\
\hline $\operatorname{MRLM}(\mathrm{db} 8, L=2)$ & 600 & 93.91 & 84.29 & 85.58 \\
\hline MRLM (coif1, $L=2$ ) & 600 & 92.63 & 88.14 & 90.71 \\
\hline \multicolumn{5}{|l|}{ VisTex dataset } \\
\hline $\operatorname{MRLM}(\mathrm{db} 1, L=2)$ & 600 & 62.50 & 45.50 & 44.50 \\
\hline $\operatorname{MRLM}(\mathrm{db} 4, L=2)$ & 600 & 63.00 & 47.00 & 44.50 \\
\hline $\operatorname{MRLM}(\mathrm{db} 8, L=2)$ & 600 & 67.50 & 41.50 & 46.00 \\
\hline MRLM (coif1, $L=2)$ & 600 & 61.00 & 48.00 & 46.00 \\
\hline MRLM (coif4, $L=2)$ & 600 & 65.00 & 44.00 & 44.00 \\
\hline
\end{tabular}

Better results are made bold.

Table 8

Classification results for three level Multi-resolution Laws' Masks (MRLM) for 25 number of different masks.

\begin{tabular}{|c|c|c|c|c|}
\hline \multirow[t]{2}{*}{ MRLM with different wavelet basis } & \multirow[t]{2}{*}{ Number of features } & \multicolumn{3}{|c|}{ Classification accuracy } \\
\hline & & Mean & Absolute mean & Standard deviation \\
\hline \multicolumn{5}{|l|}{$\overline{\text { Brodatz dataset }}$} \\
\hline $\operatorname{MRLM}(\mathrm{db} 4, L=3)$ & 900 & 87.50 & 82.69 & 87.82 \\
\hline $\operatorname{MRLM}(\mathrm{db} 8, L=3)$ & 900 & 89.42 & 79.49 & 81.41 \\
\hline MRLM (coif $1, L=3$ ) & 900 & 88.46 & 83.33 & 86.86 \\
\hline \multicolumn{5}{|l|}{ VisTex dataset } \\
\hline $\operatorname{MRLM}(\mathrm{db} 1, L=3)$ & 900 & 58.00 & 46.50 & 43.00 \\
\hline $\operatorname{MRLM}(\mathrm{db} 4, L=3)$ & 900 & 61.00 & 48.00 & 44.00 \\
\hline $\operatorname{MRLM}(\mathrm{db} 8, L=3)$ & 900 & 61.00 & 46.00 & 47.00 \\
\hline MRLM (coif1, $L=3$ ) & 900 & 60.00 & 52.00 & 47.50 \\
\hline MRLM (coif $4, L=3)$ & 900 & 57.00 & 47.50 & 46.00 \\
\hline
\end{tabular}

Better results are made bold. 
Performance of first level MRLM method with 25 nos of mask for vistex dataset

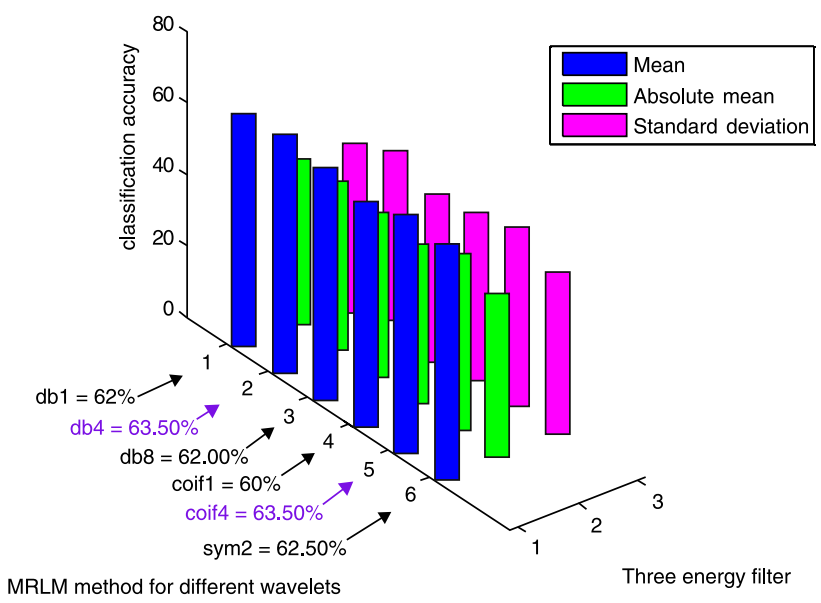

Fig. 14. MRLM (25 masks, $L=1)$ for VisTex dataset.

Performance of second level MRLM method with 25 nos of mask for vistex dataset

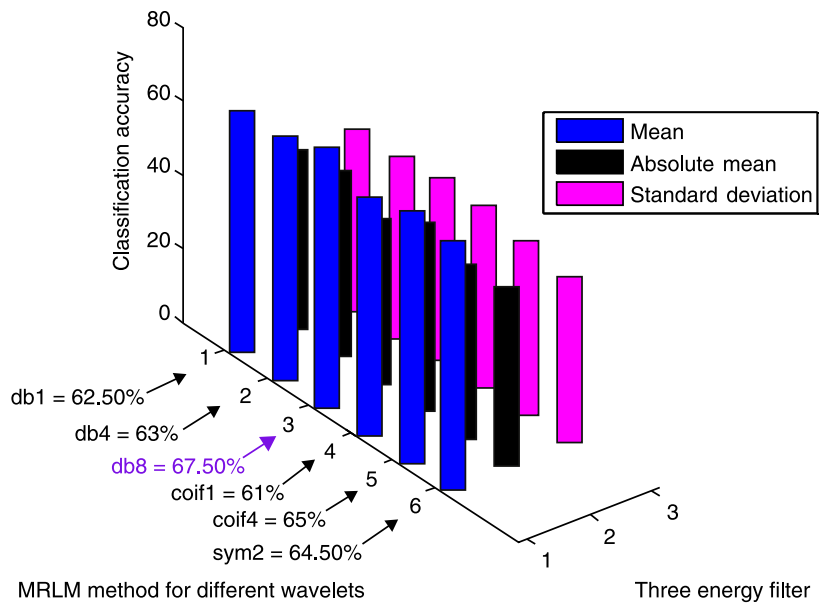

Fig. 15. MRLM (25 masks, $L=2$ ) for VisTex dataset.

Performance of third level MRLM method with 25 nos of mask for vistex dataset

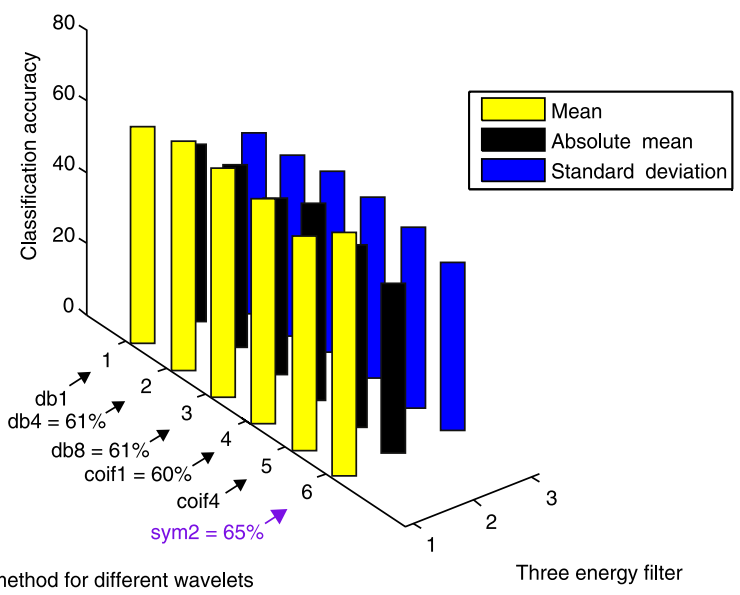

Fig. 16. MRLM (25 masks, $L=3$ ) for VisTex dataset. on Brodatz database. On the other hand, for VisTex database rise in classification accuracies are observed up to the second level of decomposition. Afterwards, the classification accuracies are reduced for some wavelets at the third level of decomposition. It has been observed that for both the databases there is a reduction in classification accuracy at the higher levels of image decomposition. The reason is that DWT create low-resolution images that do not preserve qualitative visual information.

\section{Conclusion}

The main feature of dyadic wavelet transform is multiresolution representation of images. By using the wavelets, the given images can be analyzed at various levels of resolution in both spatial and frequency domain. Wavelets seem to be effective for the analysis of textures recorded with different resolutions. Laws' masks method is a filtering method in spatial domain to extract texture features. In this paper, taking into account the aforementioned advantages of both the methods, we investigated the texture classification problem with combination of dyadic wavelet transform and Laws' masks. The proposed method performs better than the individual traditional methods. However, the results do not show any improvement at the higher levels of decomposition. The reason is that at higher level DWT produces low-resolution images that do not preserve qualitative visual information.

\section{Conflict of interest}

The authors have no conflicts of interest to declare.

\section{References}

Ade, F. (1983). Characterization of textures by eigenfilters. Signal Processing, $5(5), 451-457$.

Arivazhagan, S., \& Ganesan, L. (2003). Texture classification using wavelet transform. Pattern Recognition Letters, 24(9), 1513-1521.

Bajcsy, R., \& Lieberman, L. (1976). Texture gradient as a depth cue. Computer Graphics and Image Processing, 5(1), 52-67.

Brodatz, P. (1966). Textures. A photographic album for artists and designers. New York, USA: Dover Publications, Inc.

Cross, G. R., \& Jain, A. K. (1983). Markov random field texture models. IEEE Transactions on Pattern Analysis and Machine Intelligence, 5(1), 25-39.

de Siqueira, F. R., Schwartz, W. R., \& Pedrini, H. (2013). Multi-scale gray level co-occurrence matrices for texture description. Neurocomputing, 120, 336-345.

Dong, Y., \& Ma, J. (2013). Feature extraction through contourlet subband clustering for texture classification. Neurocomputing, 116, 157-164.

Du Buf, J. H., Kardan, M., \& Spann, M. (1990). Texture feature performance for image segmentation. Pattern Recognition, 23(3-4), 291-309.

Elnemr, H. A. (2013). Statistical analysis of law's mask texture features for cancer and water lung detection. International Journal of Computer Science Issues, 10(6), 196-202.

Ertugrul, O. F. (2014). Adaptive texture energy measure method. International Journal of Intelligent Information Systems, 3(2), 13-18.

Florindo, J. B., Assirati, L., \& Bruno, O. M. (2015). Enhancing texture descriptors by a neighbourhood approach to the non-additive entropy. Digital Signal Processing, 44, 14-25.

Haralick, R. M. (1979). Statistical and structural approaches to texture. Proceedings IEEE, 67(5), 786-804. 
Haralick, R. M., \& Shapiro, L. G. (1992). Computer and Robot Vision. Boston, MA, USA: Addison Wesley Publishing Co.

Harwood, D., Subbarao, M., \& Davis, L. S. (1985). Texture classification by local rank correlation. Computer Vision, Graphics, and Image Processing, 32(3), 404-411.

Harwood, D., Ojala, T., Pietikainen, M., Kelman, S., \& Davis, L. (1995). Classification by centre-symmetric auto-correlation using Kullback discrimination of distributions. Pattern Recognition Letters, 16(1), 1-10.

Jain, A. K., \& Farrokhnia, F. (1991). Unsupervised segmentation using Gabor filters. Pattern Recognition, 24(12), 1167-1186.

Kashyap, R. L., \& Khotanzed, A. (1986). A model based method for rotation invariant texture classification. IEEE Transactions Pattern Analysis Machine Intelligence, 8(4), 472-481.

Laine, A., \& Fan, J. (1993). Texture classification by wavelet packet signatures. IEEE Transactions on Pattern Analysis and Machine Intelligence, 15(11), 1186-1191.

Laws, K. I. (1979). Texture energy measures. In Proc. image understanding workshop (pp. 47-51).

Laws, K. I. (1980a). Textured image segmentation. Image Processing Institute, University of Southern California, IPI Report 940., 186 p.

Laws, K. I. (1980b). Rapid texture identification. Proceedings of the SPIE, 238, 376-381.

Li, S., \& Shawe-Taylor, J. (2005). Comparison and fusion of multiresolution features for texture classification. Pattern Recognition Letters, 26(5), 633-638.

Mallat, S. G. (1989a). A theory for multiresolution signal decomposition: The wavelet representation. IEEE Transaction on Pattern Analysis Machine Intelligence, 11(7), 674-693.

Mallat, S. G. (1989b). Multifrequency channel decomposition of images and wavelet models. IEEE Transaction Acoustic Speech Signal Processing, 37(12), 2091-2110.

Manjunath, B. S., \& Ma, W. Y. (1996). Texture features for browsing and retrieval of large image data. IEEE Transaction Pattern Analysis Machine Intelligence, 18(8), 837-849.

Mao, J., \& Jain, A. K. (1992). Texture classification and segmentation using multi-resolution simultaneous autoregressive models. Pattern Recognition, 25(2), 173-188.

Murala, S., \& Wu, Q. J. (2014). Expert content-based image retrieval system using robust local patterns. Journal of Visual Communication and Image Representation, 25(6), 1324-1334.

Ng, I., Tan, T., \& Kittler, J. (1992). On local linear transform and Gabor filter representation of texture. In Proceedings. 11th IAPR International Conference on Pattern Recognition, Vol. III. Conference C: Image, Speech and Signal Analysis (pp. 627-631). IEEE.

Ojala, T., Pietikainen, M., \& Harwood, D. (1996). A comparative study of texture measures with classification based on feature distributions. Pattern Recognition, 29(1), 51-59.

Ojala, T., Pietikainen, M., \& Nisula, J. (1996). Determining composition of grain mixtures by texture, classification based on feature distributions. International Journal of Pattern Recognition Artificial Intelligence, 10(1), $73-82$.

Ojala, T., Pietikainen, M., \& Maenpaa, T. (2002). Multiresolution gray-scale and rotation invariant texture classification with local binary patterns. IEEE Transactions on Pattern Analysis and Machine Intelligence, 24(7), 971-987.
Pietikainen, M., Rosenfled, A., \& Davis, L. S. (1983). Experiments with texture classification using averages of local pattern matches. IEEE Transactions on Systems, Man and Cybernetics, 13(3), 421-426.

Pikaz, A., \& Averbuch, A. (1997). An efficient topological characterization of gray-level textures, using a multiresolution representation. Graphical Models and Image Processing, 59(1), 1-17.

Qi, X., Shen, L., Zhao, G., Li, Q., \& Pietikainen, M. (2015). Globally rotation invariant multi-scale co-occurrence local binary pattern. Image and Vision Computing, 43, 16-26.

Qiao, Y. L., Zhao, C. H., \& Song, C. Y. (2009). Complex wavelet based texture classification. Neurocomputing, 72(16), 3957-3963.

Rachidi, M., Marchadier, A., Gadois, C., Lespessailles, E., Chappard, C., \& Benhamou, C. L. (2008). Laws' masks descriptors applied to bone texture analysis: An innovative and discriminant tool in osteoporosis. Skeletal Radiology, 37(6), 541-548.

Sharma, M., \& Singh, S. (2001, November). Evaluation of texture methods for image analysis. In Intelligent information systems conference, the seventh Australian and New Zealand 2001 (pp. 117-121). IEEE.

Simoncelli, E. P., \& Freeman, W. T. (1995). The steerable pyramid: A flexible architecture for multi-scale derivative computation. IEEE International Conference on Image Processing, 3, 444-447.

Singh, S., \& Sharma, M. (2001). Texture analysis experiments with Meastex and Vistex Benchmarks. In Advances in pattern recognition (ICAPR). pp. 419-426. Springer.

Susan, S., \& Hanmandlu, M. (2013). A non-extensive entropy feature and its application to texture classification. Neurocomputing, 120, 214-225.

Tang, Z., Su, Y., Er, M. J., Qi, F., Zhang, L., \& Zhou, J. (2015). A local binary pattern based texture descriptors for classification of tea leaves. Neurocomputing, 168, 1011-1023.

Unser, M. (1986). Local linear transforms for texture measurements. Signal Processing, 11(1), 61-79.

Unser, M. (1995). Texture classification and segmentation using wavelet frames. IEEE Transaction Image Processing, 4(11), 1549-1560.

Vidya, M., Ramón, V., \& Praveen, K. (2000). Texture classification using logical operators. IEEE Transactions on Image Processing, 9(10), 1693-1703.

MIT Media Lab, Vision Texture - VisTex Database. (1995). http://vismod.media.mit.edu/vismod/imagery/VisionTexture/vistex.html.

Xiong, H., Zhang, T., \& Moon, Y. S. (2000). A translation- and scale-invariant adaptive wavelet transform. IEEE Transactions on Image Processing, 9(12), 2100-2108.

Yadav, A. R., Anand, R. S., Dewal, M. I., \& Gupta, S. (2015a). Multiresolution local binary pattern variants based texture feature extraction technique for efficient classification of microscopic images of hard wood species. Applied Soft Computing, 32, 101-112.

Yadav, A. R., Anand, R. S., Dewal, M. I., \& Gupta, S. (2015b). Gaussian image pyramid based texture features for classification of microscopic images of hardwood species. Optik, 126(24), 5570-5578.

Yuan, F. (2014). Rotation and scale invariant local binary pattern based on high order directional derivatives for texture classification. Digital Signal Processing, 26, 142-152.

Zhang, Z., Wu, Q., Zhuo, Z., Wang, X., \& Huang, L. (2015). Wavelet transform and texture recognition based on spiking neural network for visual images. Neurocomputing, 151, 985-995. 